\title{
diversity of the species
}

3

4 Bárbara Hufnagel ${ }^{1 *}$, barbara.hufnagel@supagro.fr

$5 \quad$ Alexandre Soriano ${ }^{1}$, alexandre.soriano@supagro.fr

$6 \quad$ Jemma Taylor ${ }^{2}$, j.taylor2@kew.org

$7 \quad$ Fanchon Divol ${ }^{1}$, fanchon.divol@supagro.fr

8 Magdalena Kroc ${ }^{3}$, mkro@igr.poznan.pl

9 Heather Sanders ${ }^{4}$, heather.sanders@secure-harvests.com

10 Likawent Yeheyis ${ }^{5}$, likawenty@yahoo.com

11 Matthew Nelson ${ }^{2,6}$, matthew.nelson@csiro.au

12 Benjamin Péret ${ }^{1 *}$, benjamin.peret@supagro.fr

13

141 BPMP, Univ Montpellier, CNRS, INRAE, Institut Agro, Montpellier, France

152 Royal Botanic Gardens, Kew, UK

163 Institute of Plant Genetics Polish Academy of Sciences, Poznan, Poland

174 Secure Harvests, Bradford on Avon, UK

185 Amhara Agricultural Research Institute, Bahir Dar, Ethiopia

196 CSIRO, Perth, Australia

* $\quad$ Corresponding authors 
25 Background: White lupin is an old crop with renewed interest due to its seed high 26 protein content and high nutritional value. Despite a long domestication history in the 27 Mediterranean basin, modern breeding efforts have been fairly scarce. Recent sequencing of its genome has provided tools for further description of genetic resources but detailed characterization is still missing.

30 Results: Here, we report the genome sequencing of several accessions that were 31 used to establish a white lupin pangenome. We defined core genes that are present 32 in all individuals and variable genes that are absent in some and may represent a 33 gene pool for stress adaptation. We believe that the identification of novel genes, 34 together with a more comprehensive reference sequence, represents a significant 35 improvement of the white lupin genetic resources. As an example, we used this 36 pangenome to identify selection footprints and to provide a candidate gene for one of 37 the main QTLs associated with late flowering in Ethiopian lupin types. A 686 38 nucleotide deletion was identified in exon 3 of the LaFTa1 (Lupinus albus Flowering 39 Time a1) gene that suggests a molecular origin for this trait of importance, defining the need for vernalization in some lupins.

41 Conclusions: The white lupin pangenome provides a novel genetic resource to 42 better understand how domestication has shaped the genomic variability amongst 43 this crop. It will be of major importance for breeders to select new breeding traits and 44 incorporate them into new, more efficient and robust cultivars in order to face a 45 growing demand for plant protein sources, notably in Europe.

47 Keywords: White lupin, pangenome, flowering time, domestication, plant diversity. 
White lupin (Lupinus albus L.) is a pulse whose domestication started about

503000 - 4000 years ago in the Mediterranean region [1]. It is cultivated for its seeds

51 that contain high levels of proteins and are used both for food and feed [2]. The wild

52 forms of the species can only be found in the Balkan region and evidence of its

53 earliest use as a green manure and grain crop come from that same region [3]. Early

54 Greek farmers selected larger seeds and white flowers, and presumably soft-

55 seededness (water permeable seeds) was the earliest domestication trait. Greek and

56 Roman literature suggests that seed indehiscence (i.e. resistance to pod shattering)

57 had not yet been incorporated by the first century A.D. [4].

Wild collections and landraces of white lupin contain high levels of

quinolizidine alkaloids that accumulate in the seed, resulting in a bitter taste and

possible toxicity. Lysine-derived alkaloids are characteristic of the Genistoids [5-7], a

61 monophyletic basal clade belonging to the Fabaceae family. Traditionally these bitter

62 compounds are removed from white lupin seeds by soaking in water, a practice that

63 is still carried out today across the Mediterranean and Nile regions [1]. However, this

64 is uneconomic on a broad-scale, which motivated the identification of low alkaloid

65 mutants in Germany in the 1930s, aided by advances in chemistry [4]. Modern

66 cultivars of white lupin incorporate low alkaloid genes, hence the term 'sweet' lupins.

67 Breeding efforts have rarely been intensive or sustained over long periods. As

68 a result, white lupin yields remain low and highly variable, in comparison to similar

69 pulses like soybean for which important breeding efforts have been made

70 internationally. Although white lupin cultivation represents a promising crop for

71 Europe, in a political context aiming towards plant protein independence, the lack of

72 well characterised genetic resources has been hampering a fast deployment of white

73 lupin as an alternative crop to soybean imports. The recent sequencing of white lupin 
74 genome [8,9] demonstrated a resurgence of interest for this "old" crop. We believe

75 that white lupin intragenomic diversity might reflect the early traces of its slow and 76 sporadic domestication history.

77 Here we report a pangenome for white lupin that reveals important aspects of 78 the species diversity, single nucleotide polymorphisms (SNPs) and gene presence79 absence variations (PAVs). We construct a species pangenome consisting of 'core' 80 genes that are present in all individuals and 'variable' (soft-core or shell) genes that 81 are absent in some individuals [10,11]. Building on this comprehensive dataset, we 82 were able to identify a deletion in the QTL region associated with late flowering in 83 Ethiopian white lupins. The deleted gene is a homolog of the FT (Flowering Time) gene, suggesting that this deletion is at the origin of the need for vernalization in these accessions. Our analyses provide new perspectives on white lupin intraspecies diversity and domestication history.

87

\section{RESULTS}

\section{De novo assembly and pangenome construction}

We gathered a set of 39 white lupin accessions, including 25 modern cultivars,

9110 landraces and 4 wild accessions from 17 countries (Supplementary Table 1).

92 Genome sequence of 15 out of these accessions was available from a previous 93 report [8], whereas 24 accessions have been sequenced within this study to obtain 94 broader species representation. Short-read sequences have been assembled de 95 novo for each accession (28.5x mean depth, 150 bp pair-end, Supplementary Table $962)$.

97 The de novo assembly for each accession produced a total of $14.9 \mathrm{~Gb}$ of 98 contigs longer than 500 base pairs (bp) with an N50 value (the minimum contig 
99 length needed to cover $50 \%$ of the assembly) of 24,475 bp. These de novo

100 assemblies showed a mean complete BUSCOs score of $96.3 \%$, a value similar to the

101 AMIGA reference genome (97.7\%). Assembly completeness assessed by BUSCO

102 was higher than 91.7\%, for all accessions and in case of three accessions (Kiev,

103 P27174 and Magnus) the score was similar to the reference genome (Fig. 1a).

104 The pangenome was built using the iterative mapping and assembly

105 approach, in a similar strategy used to generate the Brassica oleracea [10] and

106 tomato [12] pangenomes. The assembly of $L$. albus reference genome based on

107 AMIGA accession is $450,972,408$ bp size with 38,258 predicted protein-coding genes

108 [8]. All de novo assembled contigs were compared with the reference genome to

109 identify previously unknown sequences. A total of $270 \mathrm{Mb}$ of nonreference sequence

110 with identity $<90 \%$ to the reference genome was obtained. After pangenome

111 construction and removal of contaminants and overly repetitive sequences, we

112 assembled an additional 3,663 scaffolds, with a length greater than 2,000 bp, for a

113 total length of $11,733,253 \mathrm{bp}$. Using a threshold of a minimum 10x coverage, we

114 identified 178 newly predicted protein-coding genes, among which 61 could be

115 annotated with gene ontology (GO) terms or Pfam domains (Supplementary Dataset

116 1). The white lupin pangenome, including reference and nonreference genome

117 sequences, had a total size of $462,705,661$ bp and contained 38,446 protein-coding

118 genes. The total size of the constructed pangenome is compatible with nuclear DNA

119 content estimates based on flow cytometry [13] which suggests that it represents the

120 complete genome sequence of the species. We added to the White Lupin Genome

121 portal (www.whitelupin.fr) dedicated user-friendly tools for the exploitation of the

122 pangenome, such as a BLAST tool for individual accessions, download of specific

123 regions of accessions and a genome browser mapping all the variants. 


\section{Core and variable genes}

126 The presence or absence of each protein-coding gene was predicted for each

127 of the 39 accessions based on the mapping of reads from each accession to the

128 pangenome assembly using SGSGeneLoss [14]. Likewise to other plants

129 pangenomes [10,12,15-18], we categorized genes in the white lupin pangenome

130 according to their presence frequencies, using Markov clustering in the

131 GET_HOMOLOGUES-EST pipeline [19]. The majority of the genes, 32,068 (78.5\%),

132 are core genes shared by all the 39 accessions; 6,046 soft-core (14.8\%), being

133 absent in at least one accession; and 8,776 (21.4\%) are shell, present in 2-38

134 accessions (Fig. 1b). The size of the pangenome expanded with each additional

135 accession to 38,443 genes, and extrapolation leads to a predicted pangenome size

136 of $40,844+/-289$ genes (Figure $1 b)$.

137

138 Single-nucleotide polymorphism detection and annotation

139 To capture and broadly characterize white lupin diversity we applied a strict

140 SNP identification pipeline, using GATK 4.1.0.0. A total of 9,442,876 raw SNPs were

141 identified, 806,740 of which were recognized in the newly assembled pangenome

142 scaffolds. After filtering, 3,527,872 SNPs were retained in the 39 accessions,

143 corresponding to a rate of 1 variant every 127 bp (Supplementary Figure S1). The

144 majority $(85.8 \%)$ of the high-quality variants are SNPs $(3,027,761)$ and the other

145501,111 variants detected are insertions and deletions (Fig. 1c - blue). Most variants

146 (59.3\%) are distributed on intergenic regions, 7.1\% are within introns and only $1.9 \%$

$147(96,576)$ of the variants are located in exons (Fig. 1c - red). From the variants

148 present in the CDS region 4,725 showed potentially large effects by causing start 
149 codon changes, premature stop codons or elongated transcripts, and 50,478 are

150 considered to produce a moderate effect by leading to codon changes in annotated

151 genes. The frequency of these missense SNPs in the core gene set was one each

$1524.26 \mathrm{~kb}$, which was lower than the variable gene set, with a rate of one for $1.84 \mathrm{~kb}$.

153 The rest of the variants lead to synonymous changes in proteins (low effect variants)

154 or modifiers, causing changes outside the coding regions (Fig. 1c - green).

155 Collectively, this comprehensive dataset of the genome variation of white lupin

156 provides a resource for biology and breeding of this species.

157

158 Population structure

159 To establish a phylogenetic benchmark for the analysis of the pangenome, we

160 built a consensus maximum likelihood tree (Fig. 2a) to infer the phylogenetic

161 relationships for these $L$. albus accessions using the complete set of 3.5 M SNPs

162 described above. This phylogenetic tree clustering supported six clades, which

163 exhibited distinctive geographic origin and distinctive botanical features. In the Type

1641 are grouped accessions with early flowering traits, including the Chilean

165 agrogeotypes, and German and French accessions used in breeding programs. This

166 group also included the widely used cv. Kiev Mutant, which was generated by

167 mutagenesis techniques with the intention to induce early flowering, and the

168 accessions that are derived forms of it (Primorsky and Dieta, [3]). Type 2 is also

169 composed by accessions with early flowering, anumber of which have characteristics

170 of Polish agroecotypes described by Kurlovich [3] and are adapted to grow in Eastern

171 Europe. One of the most representative accessions of this group is the cv. Kalina [3],

172 an old cultivar created in the Polish breeding program sharing similar genetic

173 background with the broadly used cultivar Start. Interestingly, Start is reported to 
174 carry different early-flowering genes than Kiev Mutant [20]. Type 2 also comprises

175 two landraces with from Syria and Israel/Palestine. Type 3 encompasses autumn-

176 sown genotypes with strong vernalisation requirement and dwarf phenotype from the

177 French breeding program, and the Algerian landrace ALB01. Algerian landraces are

178 also reported to have a strong need of vernalization [3]. Type 4 comprises landraces

179 from Iberian and Apennine Peninsula together with the described thermoneutral

180 cultivars (i.e. Neutra, [2]). Type 5 is composed only by Ethiopian landraces and Wild

181 group is composed by the four "graecus-type" accessions of the panel, all presenting

182 small black-speckled seeds and non-domesticated traits (hard seeds and shattering

183 pods).

184 We examined genetic structure by performing a Bayesian model-based

185 clustering analysis and found that the six population groups matched the maximum-

186 likelihood tree (Fig. 2b). This presented evidence of significant admixture in some

187 lines and a weak population structure, a pattern already seen in other studies of $L$.

188 albus [21]. This weak population structure is also seen through the population-

189 differentiation statistic $\left(F_{S T}\right)$. The $F_{S T}$ value between all six groups were 0.27 ,

190 however, $F_{\text {ST }}$ between Type 1 and Type 2 are low as 0.086, and Type 4 and Wild

191 have an $\mathrm{F}_{\mathrm{ST}}$ of 0.092 . Indeed, regarding the Bayesian model, in scenarios dividing

192 the accessions in 4 or 5 sub-populations (Fig $2 b, K=4$ and $K=5$ ), accessions from

193 Type 4 are merged with the Wild group. On the other hand, Type 5 showed a strong

194 differentiation from the other groups, with $\mathrm{F}_{\mathrm{ST}}$ values ranging from 0.34 to 0.46 , with

195 Type 4 and Type 3, respectively, which is corroborating with previous studies [22].

196 Principal component analysis reinforced the similarity among some groups (Fig. 2c).

197 The two first principal components explain $65.9 \%$ of genotypic variance and it is

198 highlighting the overlap among certain groups, in particular, Type 1 and Type 2. 
199 Differentiation of genetic diversity between the 6 groups was investigated 200 further through analysis of decay of linkage disequilibrium (LD, Fig. 2d). The decay of

201 LD with physical distance between SNPs to half of the maximum values occurred at $2023.85 \mathrm{~Kb}\left(r^{2}=0.38\right)$, consistent with a high level of diversity and partially outcrossing 203 mode of reproduction in this species [23]. Type 4 group also showed a fast LD decay 204 of $5.7 \mathrm{~Kb}\left(\mathrm{r}^{2}=0.40\right)$ and Type $1-3$ groups have an average LD decay of $10.5 \mathrm{~Kb}$. 205 Wild group showed a slower LD decay $\left(38.1 \mathrm{~Kb}, \mathrm{r}^{2}=0.39\right)$ when comparing with the 206 other white lupin groups, presumably an effect of the small number of wild 207 accessions in the analysis. Nevertheless, these LD decay levels can still be 208 considered fast compared with other plant species, for example rice $(\sim 75-150 \mathrm{~Kb}$, 209 [24]), soybean ( 340-580 Kb, [25]) or wheat ( 7-12.4 Mb, [26]), also self-pollinated 210 crops. The Type 5 group (Ethiopian landraces) only reached half of its LD decay after $2111.5 \mathrm{Mb}$, reinforcing the high similarity of its accessions and a possible genetic 212 isolation of this group [21]. The average nucleotide diversity $\pi$ per site [27] showed 213 that diversity was five times lower in Type 5 group $(\pi=0.068)$ compared to the 214 general nucleotide diversity $(\pi=0.372)$. While the Wild group, although is also

215 composed of only four accessions, showed a nucleotide diversity $\pi=0.402$.

216

217 Protein-coding genes presence and absence characterization

218 Presence and absence variants (PAVs) are an important type of structural 219 variation and play an important role in shaping genomes, therefore contributing to 220 phenotypic diversity [28]. The construction of a white lupin pangenome allowed 221 identification of 1195 PAVs, representing protein-coding genes that are absent in at 222 least one of the accessions, being 1132 genes from the reference genome and 63 223 from the newly identified genes (Supplementary Dataset 2-3). We further examined if 
224 the phylogenetic groups have an influence in the number of PAVs and if the PAVs

225 are homogeneous within the groups (Fig. 3a-c). The wild accessions have a 226 significatively higher number of newly identified genes, with the accessions

227 GRAECUS and GR38 only missing 4 of them. The four wild accessions share 157 228 out of the 178 new-identified genes in the pangenome (Fig. 3a).

229 The number of missing genes within individual genomes ranges from 45 230 (AMIGA - Type 1) to 348 genes (GRC5262B - Wild). Each group shares a median of 23131 common lost genes amongst all its accessions and a total of 103 genes are 232 absent in at least one accession of each group (Fig. 3b). There are 137 genes that 233 have been exclusively lost within accessions of the Wild group, however only 30 234 genes are shared among all the graecus accessions. On the other hand, genomes of 235 Ethiopian landraces (Type 5) share a total of 118 common missing genes, amongst 23639 are unique for this group. Remarkably, for this group there is a concentration of 237 lost genes on Chr17. This includes a set of 9 tandem duplicated genes covering a 238 region of $120 \mathrm{~Kb}$ (Supplementary Fig. 2). They are annotated as "Putative ferric239 chelate reductase (NADH)" homologs of Arabidopsis gene FRO2, known for its role 240 of iron uptake by the roots under stress condition [29].

241 Checking the position of the PAVs on the chromosomes we could identify 242 some peculiarity regarding the PAVs within the groups. For example, on Chr13 there 243 is a concentration of PAVs in the region of $5-10 \mathrm{Mb}$ that are missing from most 244 accessions of Type 2-5 and Wild, but are present in the genomes of most Type 1 245 members. Similar pattern happens in the 3.6-6.4 Mb region of Chr04. Chr23 has the 246 highest number of PAVs (78), a common feature of all the groups.

247 Functional analysis of PAVs suggests enrichment of GO terms as "integral 248 component of membrane" (GO:0016021) and "oxidation-reduction process" 
249 (GO:0055114) (Fig. 3d, supplementary Fig. 3 and Supplementary Data 2). These

250 suggest an enrichment of genes and gene families coding for membrane receptors

251 proteins or membrane transporters. Other GO terms suggest that some of the genes

252 may be involved in cell wall remodeling ("cell wall" - GO:0005618; "cell wall

253 organization" - GO:0071555). Genes with these functions are frequently linked to

254 biotic and abiotic stress responses [30,31]. PAV genes related to abiotic and biotic

255 stress responses have been observed in several plant species $[15,17,18,32-35]$ and

256 these may reflect the evolution for adaptive traits related for each agroecotype.

257 Moreover, the presence/absence of these stress-response related genes may also

258 be partially due to whole-genome triplication event on white lupin genome [8], which

259 caused an overlapping roles in various loci.

260

261 Footprints of selection and alleles identification in candidate genes

262 To demonstrate the power of white lupin pangenome to address basic 263 research questions, we used it to detect possible footprints of selection and to 264 identify alleles in candidate genes underlying major QTLs. Firstly, to examine 265 potential selective signals during white lupin domestication and breeding, we 266 scanned white lupin genome searching for regions with marked reductions in 267 nucleotide diversity (Fig. 4).

268 The domestication and breeding efforts in white lupin have focused in 269 searching for accessions with reduced seed alkaloid content, reduced time to flower 270 as well as excessive indeterminate branching. Therefore, we combined Type 1 and 271 Type 2 accessions, that are spring types and went to a more intense breeding 272 process and compared them with Type 3 and Type 4 accessions, that are winter 273 types (Fig. 4a). A selective sweep affecting only the spring white lupin accessions 
274 would be expected to leave a typical low-polymorphism and high-divergence signal 275 around the region of the selected genes. We measured the sweep on the nucleotide 276 diversity ( $\pi$ value [27]), by comparing the two groups ( $\pi$ Winter/mSpring) over 250-kb 277 windows. We identified 167 putative selection sweeps associated to the breeding of 278 the spring accessions ( $\pi$ Winter/mSpring $>2.101$ ). We observed that some of the 279 peaks co-localized with previously reported white lupin QTLs for flowering time and 280 alkaloid content [36,37]. The same pattern was observed when checking for the 281 divergence of the gene pool between these two groups along the chromosomes (Fst, 282 Supplementary Fig. 4a).

Interestingly, other peaks with higher sweeps of diversity are present, 284 indicating that other genomic regions may be implicated with these traits and may 285 carry other important genes of these pathways. Furthermore, they highlight specific 286 genomic regions of spring accessions that have been selected during domestication 287 and breeding. For instance, we checked for orthologs of domestication genes from 288 the close relative narrow-leafed lupin and found that the gene Lalb_Chr12g0203121, a homolog of a candidate gene for the reduced pod shattering locus tardus 290 (Lup002448, [38]), is co-localized with a sweep peak on Chr12.

291 The reported white lupin QTLs were identified in a recombinant inbred line 292 (RIL) mapping population derived from the cross between Kiev Mutant (Type 1) and 293 the Ethiopian landrace P27174 (Type 5). Thereupon, we checked the sweep of 294 diversity between all accessions compared to Ethiopian accessions, T5

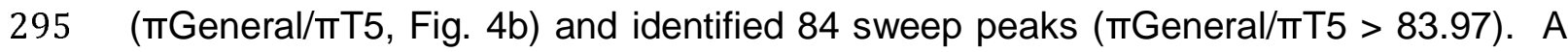
296 similar trend of co-localization of the QTL peaks were observed, with steep peaks 297 around the QTL regions. Interestingly, the region corresponding the QTL pauper did 298 not show a peak, being far below the statistical significance threshold. This indicates 
299 that the two groups have similar level of nucleotide diversity in this region 300 (Supplementary Fig. 4b). It can be explained by the above-mentioned similarities 301 among accessions of group T5 and that many of the modern accessions carry the 302 low alkaloid alleles for the pauper region. However, although this region showed a 303 similar nucleotide diversity between the two groups, it presented a high genetic 304 variance, with a median FST of 0.94 for the region (Fig. 4c).

305 In another approach to demonstrate the power of white lupin pangenome, we 306 used its assembly to identify a candidate gene underlying a major QTL and describe 307 the associated allelic diversity. Chromosome 2 is the location of an important QTL 308 associated with early flowering white lupins. We used the protein sequences of 309 Lupinus angustifolius that have been previously mapped in syntenic regions of the 310 these QTLs [39] to perform an homology search against the pangenome. we 311 identified the gene LaFTa1 (Lalb_Chr02g0156991), a homolog of the gene LanFTa1

312 (Lup21189) mapped on this QTL region. The white lupin LaFTa1 313 (Lalb_Chr02g0156991) was annotated as "Putative phosphatidylethanolamine314 binding protein" (PEBP) in the reference genome. The FT proteins belonging to the 315 PEBP family are the key control points of the flowering time in plants. The LaFTa1 316 gene presented a deletion of 686 on the third intron that is present only on Type 5 317 accessions, that have late flowering phenotypes (Fig. 5a-b and Supplementary Fig. 318 5). Indeed, one of the parents of this QTL mapping population belongs to this group 319 (P27174). It is reported that changes in FT promoter and introns can alter FT 320 expression in response to photoperiod and vernalization, and consequently, induce 321 flowering [40]. This suggests that the identified LaFTa1 is the gene underlying this 322 QTL and that this deletion on the intron of Type 5 accessions may be contributing for 323 the late flowering pattern of this group. 


\section{DISCUSSION}

326 A pangenome is a complete set of genes for a species, including core genes

327 which are present in all individuals, and variable genes which are absent in one or

328 more individuals [41]. We generated a de novo assembly for 38 white lupin

329 accessions and, taking advantage of a good reference assembly for the species [8],

330 we constructed a $L$. albus pangenome by iteratively and randomly sampling these

331 sequenced accessions. This dataset is representative of the diversity of the species,

332 containing wild accessions, landraces and cultivars of white lupin from across their

333 respective distributions. As a result, we estimate that this white lupin pangenome

334 assembly effectively encompasses the complete sequence for the genome of the

335 species, with $462,7 \mathrm{Mb}$ sequence and containing 38,446 protein-coding genes. The

336 finding that $21.5 \%$ of genes in the pangenome exhibit varying degrees of genic

337 presence/absence variants (PAVs) highlights the diverse genetic feature of white

338 lupin and the significant improvement of the reference genome, by including genomic

339 information of other accessions and discovery of new genes. Remarkably, the white

340 lupin pangenome showed a high content of core genes (78.5\%), as compared with

341 other plant species as tomato (74.2\%, [34]), Arabidopsis thaliana (70\%,[19]), bread

342 wheat (64\%, [42]), sesame (58\%, [16]) and wild soybean (49\%, [43]), which might be

343 a reflection of its domestication history and modest breeding efforts to date.

344 The domestication of white lupin started during Bronze Age [4], and the 345 ancestral history of this species is different than other major crops such as rice,

346 maize, sorghum, tomato, and soybeans, which are more ancient [44]. The early

347 cultivated forms have the same Mediterranean distribution that its wild ancestor types

348 (graecus), which led to small adaptation or selection differences. L. albus 
349 domestication was slow with potentially centuries between acquisition of each

350 domestication trait, which may explain why there is not a more pronounced genetic

351 differentiation between wild, landrace and cultivated types [45]. This is echoed in the

352 lack of population structure presented within these accessions and in the low LD

353 extent, which generally reduce the diversity and change allele frequencies either to

354 fixation or intermediate frequencies [46]. Despite being a largely self-pollinating crop

355 (with an out-crossing rate reported as 8-10\%, [23]), white lupin showed a

356 remarkably low LD extent $(<4 \mathrm{~kb})$, even lower than the wild population of its relative,

357 narrow-leafed lupin, that showed a decay of LD after $19.01 \mathrm{~Kb}$ [47]. One distinction

358 between these closely related species is that narrow-leafed lupin is almost

359 exclusively self-pollinating and so the modest levels of outcrossing in white lupin may

360 be a key factor governing the differences in LD between these two species. Having a

361 low LD and weak population structure together mean that association mapping is

362 likely to be particularly powerful in white lupin, in contrast to the more highly

363 structured and high LD species narrow-leafed lupin, where association studies have 364 so far proved rather weak $[47,48]$.

365 Type 5 accessions, from Ethiopia, are the only group which shown a strong

366 genetic differentiation from the others, with $\mathrm{F}_{\mathrm{ST}}$ values higher than 0.3 . Such a distinct

367 separation is an evidence that the Ethiopian accessions have evolved in isolation and

368 the genetic differences are probably due to ancient founder effects. The differences

369 of Type 5 group are also highlighted by the PAVs. Together with the Wild group,

370 Ethiopian landraces carry most of the new identified genes and also miss a large

371 number of genes of the reference genome (Fig. 3). Moreover, it is a highly

372 homogeneous group, with all accessions sharing a large number of these lost genes.

373 The loss of these genes is probably an adaptive response for the local environment. 
374 For instance, the loss of the nine tandem duplicated homologue AtFRO2 on Chr17

375 might be an adaptive response to highland Ethiopian soils that are iron-rich [49]. A

376 more detailed look into the PAVs among the different groups may be useful to better

377 understand their specificities.

378 Our analysis brings a high resolution to the within-species diversity. Using the 379 pangenome dataset, we performed genome-wide comparisons of the assemblies, 380 enabling the characterization of more than 3 million complex variants, including many

381 large-effect coding variants which should be helpful in pinpointing causal variations in

382 QTLs for important traits and in future genome-wide association studies. In particular, 383 our study demonstrated that 4,725 genes were found to contain important coding 384 variation in at least one accession and might have important biological functions 385 underlying the variation of complex traits.

386 We wanted to demonstrate how a pangenome can be a useful tool to identify 387 allelic differences that are responsible for phenotypic variation. By performing a 388 genome wide analysis, we detected that nucleotide diversity were quite variable across the genome. The efforts of breeding in white lupin have been focused in combining of domestication traits such as soft and white seeds and reduced pod-

391 shattering, which were already available from ancient times, with that of reduced 392 alkaloids, increased yield and the reduction of flowering time and excessive 393 branching [45]. Looking for differences in nucleotide diversity across the genome 394 amongst breeding accessions and comparing with landraces/wild accessions, we 395 could detect some peaks of sweep of diversity. In these peaks there is an important 396 decrease of nucleotide diversity within the breeding lines and they represent marks of 397 selection (Fig. 4). In these regions were also detect a high divergence between the 398 two gene pools (Fst). However, although the sweeps of diversity co-localize with 
399 some identified QTLs for flowering time and low alkaloid content, there are other

400 higher peaks along the chromosomes. These regions should be explored in order to

401 find genes underlying phenotypic traits that have been selected directly or indirectly

402 during domestication and breeding of white lupin. For instance, white lupin is known

403 for thriving in soils with low nutrient availability by producing specialized root

404 structures called cluster roots [50]. In a previous work, we demonstrated that the

405 breeding accessions have an earlier establishment of the root system through lateral

406 and cluster root formation that was indirectly selected [8]. By looking closer in these

407 chromosome regions with low nucleotide diversity and high genetic differentiation we

408 might be able to find genes with important roles in the root architecture of white lupin.

409 Hence, integration of the information from studies of gene function and the high

410 density of variants described in this pangenome can provide a complementary

411 approach to forward genetic studies and can contribute to develop the research and

412 breeding of white lupin.

413

414 CONCLUSION

415 In summary, the white lupin pangenome comprises a wealth of information on

416 genetic variation that has yet to be fully exploited by researchers and breeders.

417 Although a there is large collection of white lupin accessions available in genebanks

418 worldwide, they barely have been explored and genetically characterized. This

419 pangenome represents a comprehensive and important resource to facilitate the

420 exploration of white lupin as a legume model for future functional studies and

421 molecular breeding.

422

423 


\section{METHODS}

\section{Genome sequences of white lupin accessions}

426 We retrieved the genome sequencing data of 15 white lupin accessions that 427 were published previously [8], including 11 modern cultivars, 1 landrace and 2 wild 428 relatives. They were sequenced using Illumina technology using paired-end $2 \times 150$ 429 bp short-reads with average sequencing depth of $45.99 \times$. It included Illumina genome 430 data of $64.47 x$ depth for the reference cultivar "AMIGA". Genome sequences of 431 additional 24 accessions were generated here, including 12 modern cultivars, 9 432 landraces and 2 wild relatives. Young leaves of individual plants were used to extract 433 genomic DNA of each accession using the QIAGEN DNeasy Plant Mini kit following 434 the supplier's recommendations. The accessions were sequenced using Illumina 435 technology using paired-end $2 \times 150$ bp short-reads (Macrogen, South Korea). It was 436 generated a total of $196.85 \mathrm{~Gb}$ of data with average sequencing depth of $19.1 \mathrm{x}$. 437 (Supplementary Table 2).

438

\section{De novo genome assembly and pangenome construction}

440 Reads were processed to trim adapters and low-quality sequences using 441 Cutadapt 1.15 [51] with parameters '--pair-filter=any -q20,20 -m 35' and the forward 442 and reverse Illumina TruSeq Adapters. The final high-quality cleaned Illumina reads 443 from each sample were de novo assembled using Spades 3.13.0 [52] with k-mer size 444 of $21,33,55,77,99,121$. The assembled contigs were then aligned to the white lupin 445 reference genome [8] (GenBank accession no.: WOCE00000000, 446 http://www.whitelupin.fr.), using the steps 7 and 8 of the EUPAN Pipeline [53], in 447 order to extract contigs that were not aligning to the reference. Then, redundancy in 448 the extracted contigs has been reduced using CD-hit 4.8.1 with default parameters. 
449 The resulting contigs were then search against the NCBI nt nucleotide database

450 using blastn 2.10 [54]. Sequences with best hits from outside the Eudicots, or

451 covered by known plant mitochondrial or chloroplast genomes, were possible

452 contaminations and were therefore removed.

453

\section{Annotation of the white lupin pangenome}

455 A custom repeat library was constructed by screening the pangenome and the 456 white lupin reference genome using RepeatModeler

457 (http://www.repeatmasker.org/RepeatModeler/), and used to screen the nonreference 458 genome to identify repeat sequences using RepeatMasker

459 (http://www.repeatmasker. org/). Contigs with more than $98 \%$ of repetitive sequences

460 were removed from the annotation pipeline. Protein-coding genes were predicted 461 from nonreference genome using MAKER2 [55]. Ab initio gene prediction was 462 performed using Augustus [56] and SNAP [57]. Augustus [58] has been previously 463 trained for white lupin as described in the documentation, and SNAP was trained for 464 two rounds based on already assembled transcriptome of white lupin, as described in 465 maker2 documentation. In addition, protein sequences of white lupin, Medicago 466 truncatula and the Viridiplantae subset of Swissprot were used as evidence. Finally, 467 gene predictions based on ab initio approaches, and transcript and protein evidence 468 were integrated using the MAKER2 pipeline. A set of high-confidence gene models 469 supported by transcript and/or protein evidence were generated by MAKER2. In 470 order to remove possible remaining contamination, all high confidence maker 471 generated protein sequences were aligned against the $\mathrm{nr}$ databses, and sequences 472 with best hits from outside Eudicots or with best hit inside chloroplastic and 
473 mitochondrial sequences were removed. Genes that matched white lupin reference

474 sequences were also removed the same way.

475 In parallel, contigs with a length superior to $2 \mathrm{~Kb}$ from the whole assembly of

476 the 39 lupin accession were annotated using the Egnep 1.5.1 pipeline [59].

477 RepeatMasker was used to detect and remove contigs constitute by more than $98 \%$

478 of known repeat sequences based on the previously built white lupin repetitive 479 element sequences database. The white lupin transcriptome [8]was used as ESTs 480 evidence, using a minimum identity percentage of $95 \%$, along with the proteome of 481 white lupin, Medicago truncatula, and the Viridiplantae subset of the swissprot 482 database, with weight of $0.4,0.3$ and 0.3 respectively. Resulting predicted proteins 483 were search against REXdb and repbase in order to remove possible transposable 484 elements. The resulting genes prediction were again scan with repeat-masker, and 485 genes composed of more than $90 \%$ of detected repetitive sequences were removed 486 from further analyses in order to control false positive.

487

488 Gene presence/absence variation and pangenome modeling

489 Reads were processed to trim adapters and low-quality sequences using 490 Cutadapt 1.15 [51] with parameters '--pair-filter=any -q20,20 -m 35' and the forward 491 and reverse Illumina TruSeq adapters. Resulting high quality reads were then aligned 492 to the pangenome using BWA-MEM [60] with default parameters. Picard tools was 493 used to remove possible PCR and optical duplicates, and reads considered as not 494 properly paired were removed using samtools view. The presence or absence of 495 each gene in each accession was determined using SGSGeneLoss [14] . In brief, for 496 a given gene in a given accession, if less than $10 \%$ of its exon regions were covered 497 by at least five reads ( $\operatorname{minCov}=5$, lostCutoff $=0.1$ ), this gene was treated as absent 
498 in that accession, otherwise it was considered present. The parameters used for the

499 new set of gene discovered in the pangenome were different: minCov $=10$ and

500 lostCutoff $=0.8$. For more precise pangenome studies taking into account all the 501 genes discovered in all the different varieties, GET_HOMOLOGUES_EST was used 502 on the whole CDS and proteome of the whole 39 varieties with parameters "- $R$ $503123545-\mathrm{P}-\mathrm{M}-\mathrm{c}-\mathrm{z}-\mathrm{A}$-t 2" to detect clusters of genes shared by at least two 504 varieties.

505

506 SNP discovery and annotation

507 Cutadapt [61] was used to remove Illumina Truseq adapters from the 508 sequencing data and to remove bases with a quality score lower than 30 , in both $5^{\prime}$ 509 and 3' end of the reads. Reads with a length lower than 35 were discarded. We then 510 used BWA-MEM version 0.7.17 [60] to map the resequencing reads from all 39 511 genotypes to the white lupin reference genome. PCR and Optical duplicates were 512 detected and removed using Picard Tools. After that, GATK 4 HaplotypeCaller tool 513 was used in emit-ref-confidence GVCF mode to produce one gvcf file per sample. 514 These files were merged using GATK Combine GVCFs. Finally, GATK 515 GenotypeGVCFs was used to produce a vcf file containing variants from all the 39 516 samples. This identified a total of 9,442,876 SNPs/indel. After filtering for minimum 517 allele frequency of 0.15 and heterozygosity frequency of $0-0.2,3,527,872$ SNPs 518 were retained for further analysis.

519

\section{Evolutionary analysis}

521 A maximum-likelihood phylogenetic tree was constructed based on 3,121,673 522 parsimony-informative SNPs with 1,000 bootstraps using IQ-TREE [62] using 
523 ModelFinder [63] option. Then, a phylogenetic tree was prepared using the iTOL v $5244.3[64]$.

$525 \quad$ Population structure based on the same set of SNPs was investigated using 526 STRUCTURE [65]. Thirty independent runs for each $\mathrm{K}$ from 1 to 15 were performed 527 with an admixture model at 50,000 Markov chain Monte Carlo (MCMC) iterations and 528 a 10,000 burn-in period. Principal component analysis using this SNP dataset was 529 performed using the function "princomp" in R (http://www.R-project.org/). The linkage 530 disequilibrium (LD) pattern was computed using PopLDdecay v3.40 [66]. LD decay 531 was measured on the basis of the $r^{2}$ value and the corresponding distance between 532 two given SNPs.

533

\section{Selective sweep analyses}

$535 \quad$ To detect genomic regions affected by domestication we used the same set of $5363,121,673$ SNPs using Tassel [67]. The level of genetic diversity ( $\pi)$ was measured 537 with a window size of 2000 SNPs and a step size of the same length, generating 538 windows of approximately $250-\mathrm{kb}$. Genome regions affected by selection or 539 domestication should have substantially lower diversity in spring white lupin (Types 1 540 and 2, mSpring) than the diversity in winter accession (Type 3 and 4, mWinter) and

541 Ethiopian accessions ( $\pi T 5)$. Windows with the top $10 \%$ highest ratios of $542 \pi$ Winter/mSpring $(\geq 2.101)$ or $\pi$ General/mCA ( $\geq 83.969)$ were selected as candidate 543 selection and domestication sweeps. The PopGenome package [68] in $R$ with its 544 sliding window method was used to calculate the interpopulation differentiation, FST. 545 Using a set of $40 \mathrm{k}$ random good-quality SNPs evenly distributed along the 25 546 chromosomes, we calculated nonoverlapping sliding-windows of 10 SNPs each. 


\section{REFERENCES}

549 1. Taylor JL, De Angelis G, Nelson MN. How Have Narrow-Leafed Lupin Genomic

550 Resources Enhanced Our Understanding of Lupin Domestication? Springer, Cham;

551 2020. p. 95-108.

552 2. Wolko B, Clements JC, Naganowska B, Nelson M, Hua'an Y. Lupinus. Kole C, 553 editor. Wild Crop Relat. Genomic Breed. Resour. Legum. Crop. Forages. Berlin, 554 Heidelberg: Springer Berlin Heidelberg; 2011.

555 3. Kurlovich BS. Lupins: Geography, Classification, Genetic Resources and 556 Breeding. Publishing House "Intan”; 2002.

557 4. Gladstones JS. Distribution, origin. taxonomy, history and importance. Lupins as

558 Crop Plants Biol Prod Uti-lization Gladstones JS, Atkins C, Hamblin J(eds) CAB Int 559 Oxon, New York. 1998. p. 1-39.

560 5. Kinghorn AD, Hussain RA, Robbins EF, Balandrin MF, Stirton CH, Evans S V. 561 Alkaloid distribution in seeds of Ormosia, Pericopsis and Haplormosia. 562 Phytochemistry. 1988;27:439-44.

563 6. van Wyk B-E. The value of chemosystematics in clarifying relationships in the 564 genistoid tribes of papilionoid legumes. Biochem Syst Ecol. 2003;31:875-84.

565 7. Wink M, Mohamed GIA. Evolution of chemical defense traits in the Leguminosae: 566 mapping of distribution patterns of secondary metabolites on a molecular phylogeny 567 inferred from nucleotide sequences of the rbcL gene. Biochem Syst Ecol. $568 \quad 2003 ; 31: 897-917$.

569 8. Hufnagel B, Marques A, Soriano A, Marquès L, Divol F, Doumas $P$, et al. High570 quality genome sequence of white lupin provides insight into soil exploration and 571 seed quality. Nat Commun. Springer US; 2020;11:492.

572 9. Xu W, Zhang Q, Yuan W, Xu F, Muhammad Aslam M, Miao R, et al. The genome 
573 evolution and low-phosphorus adaptation in white lupin. Nat Commun. Springer US;

$574 \quad 2020 ; 11: 1069$.

575 10. Golicz AA, Bayer PE, Barker GC, Edger PP, Kim H, Martinez PA, et al. The 576 pangenome of an agronomically important crop plant Brassica oleracea. Nat 577 Commun. Nature Publishing Group; 2016;7:13390.

578 11. Vernikos G, Medini D, Riley DR, Tettelin H. Ten years of pan-genome analyses. 579 Curr Opin Microbiol. 2015;23:148-54.

580 12. Gao L, Gonda I, Sun H, Ma Q, Bao K, Tieman DM, et al. The tomato pan581 genome uncovers new genes and a rare allele regulating fruit flavor. Nat Genet. $582 \quad$ Springer US; 2019;51:1044-51.

583 13. Naganowska B, Wolko B, Śliwińska E, Kaczmarek Z. Nuclear DNA content 584 variation and species relationships in the genus Lupinus (Fabaceae). Ann Bot. $585 \quad 2003 ; 92: 349-55$.

586 14. Golicz AA, Martinez PA, Zander M, Patel DA, Van De Wouw AP, Visendi P, et al. 587 Gene loss in the fungal canola pathogen Leptosphaeria maculans. Funct Integr 588 Genomics. 2015;15:189-96.

589 15. Gordon SP, Contreras-Moreira B, Woods DP, Des Marais DL, Burgess D, Shu S, 590 et al. Extensive gene content variation in the Brachypodium distachyon pan-genome 591 correlates with population structure. Nat Commun. Springer US; 2017;8:2184.

592 16. Yu J, Golicz AA, Lu K, Dossa K, Zhang Y, Chen J, et al. Insight into the evolution 593 and functional characteristics of the pan-genome assembly from sesame landraces 594 and modern cultivars. Plant Biotechnol J. 2019;17:881-92.

595 17. Zhao J, Bayer PE, Ruperao P, Saxena RK, Khan AW, Golicz AA, et al. Trait 596 associations in the pangenome of pigeon pea ( Cajanus cajan ). Plant Biotechnol J. 597 2020;pbi.13354. 
598 18. Montenegro JD, Golicz AA, Bayer PE, Hurgobin B, Lee H, Chan C-KK, et al. The 599 pangenome of hexaploid bread wheat. Plant J. 2017;90:1007-13.

600 19. Contreras-Moreira B, Cantalapiedra CP, García-Pereira MJ, Gordon SP, Vogel 601 JP, Igartua E, et al. Analysis of Plant Pan-Genomes and Transcriptomes with 602 GET_HOMOLOGUES-EST, a Clustering Solution for Sequences of the Same 603 Species. Front Plant Sci. 2017;8:1-16.

604 20. Adhikari K, Buirchell B, Yan G, Sweetingham M. Two complementary dominant 605 genes control flowering time in albus lupin (Lupinus albus L.). Plant Breed. $606 \quad 2011 ; 130: 496-9$.

607 21. Raman R, Cowley RB, Raman H, Luckett DJ. Analyses Using SSR and DArT 608 Molecular Markers Reveal that Ethiopian Accessions of White Lupin 609 (\&amp;It;i\&amp;gt;Lupinus albus\&amp;It;/\&amp;gt; L.) Represent a Unique 610 Genepool. Open J Genet. 2014;04:87-98.

611 22. Raman R, Cowley RB, Raman H, Luckett DJ. Analyses Using SSR and DArT 612 molecular markers reveal that Ethiopian accessions of white lupin (Lupinus albus L.) 613 represent a unique genepool. Open J Genet. 2014;4:87-98.

614 23. Green A, Brown A, Oram R. Determination of outcrossing rate in a breeding 615 population of Lupinus albus L. (White Lupin). Plant Breed. 1980;84:181-91.

616 24. Mather K a, Caicedo AL, Polato NR, Olsen KM, McCouch S, Purugganan MD. 617 The extent of linkage disequilibrium in rice (Oryza sativa L.). Genetics. $618 \quad 2007 ; 177: 2223-32$.

619 25. Hyten DL, Choi I-Y, Song Q, Shoemaker RC, Nelson RL, Costa JM, et al. Highly 620 Variable Patterns of Linkage Disequilibrium in Multiple Soybean Populations. 621 Genetics. 2007;175:1937-44.

622 26. Molero G, Joynson R, Pinera-Chavez FJ, Gardiner L, Rivera-Amado C, Hall A, et 
623 al. Elucidating the genetic basis of biomass accumulation and radiation use efficiency

624 in spring wheat and its role in yield potential. Plant Biotechnol J. 2019;17:1276-88.

625 27. Tajima F. Evolutionary relationship of DNA sequences in finite populations.

626 Genetics. 1983;105:437-60.

627 28. Marroni F, Pinosio S, Morgante M. Structural variation and genome complexity: is 628 dispensable really dispensable? Curr Opin Plant Biol. Elsevier Current Trends; $6292014 ; 18: 31-6$.

630 29. Connolly EL, Campbell NH, Grotz N, Prichard CL, Guerinot M Lou. 631 Overexpression of the FRO2 Ferric Chelate Reductase Confers Tolerance to Growth 632 on Low Iron and Uncovers Posttranscriptional Control. Plant Physiol. $6332003 ; 133: 1102-10$.

634 30. Osakabe Y, Yamaguchi-Shinozaki K, Shinozaki K, Tran LSP. Sensing the 635 environment: Key roles of membrane-localized kinases in plant perception and 636 response to abiotic stress. J Exp Bot. 2013;64:445-58.

637 31. Novaković L, Guo T, Bacic A, Sampathkumar A, Johnson KL. Hitting the Wall638 Sensing and Signaling Pathways Involved in Plant Cell Wall Remodeling in 639 Response to Abiotic Stress. Plants (Basel, Switzerland). MDPI; 2018;7:89.

640 32. Bayer PE, Golicz AA, Tirnaz S, Chan CKK, Edwards D, Batley J. Variation in 641 abundance of predicted resistance genes in the Brassica oleracea pangenome. Plant 642 Biotechnol J. 2019;17:789-800.

643 33. Zhou P, Silverstein KAT, Ramaraj T, Guhlin J, Denny R, Liu J, et al. Exploring 644 structural variation and gene family architecture with De Novo assemblies of 15 645 Medicago genomes. BMC Genomics. BMC Genomics; 2017;18:1-14.

646 34. Gao L, Gonda I, Sun H, Ma Q, Bao K, Tieman DM, et al. The tomato pan647 genome uncovers new genes and a rare allele regulating fruit flavor. 
648 35. Shen X, Liu ZQ, Mocoeur A, Xia Y, Jing HC. PAV markers in Sorghum bicolour:

649 genome pattern, affected genes and pathways, and genetic linkage map

650 construction. Theor Appl Genet. 2015;128:623-37.

651 36. Phan HTT, Ellwood SR, Adhikari K, Nelson MN, Oliver RP. The first genetic and 652 comparative map of white lupin (Lupinus albus L.): Identification of QTLs for 653 anthracnose resistance and flowering time, and a locus for alkaloid content. DNA 654 Res. 2007;14:59-70.

655 37. Książkiewicz M, Nazzicari N, Yang H, Nelson MN, Renshaw D, Rychel S, et al. A 656 high-density consensus linkage map of white lupin highlights synteny with narrow657 leafed lupin and provides markers tagging key agronomic traits. Sci Rep. $658 \quad 2017 ; 7: 15335$.

659 38. Plewiński P, Książkiewicz M, Rychel-Bielska S, Rudy E, Wolko B. Candidate 660 domestication-related genes revealed by expression quantitative trait loci mapping of 661 narrow-leafed lupin (Lupinus angustifolius L.). Int J Mol Sci. 2019;20:1-24.

662 39. Rychel S, Książkiewicz M, Tomaszewska M, Bielski W, Wolko B. FLOWERING 663 LOCUS T, GIGANTEA, SEPALLATA, and FRIGIDA homologs are candidate genes 664 involved in white lupin (Lupinus albus L.) early flowering. Mol Breed. 2019;39:43.

665 40. Andrés F, Coupland G. The genetic basis of flowering responses to seasonal 666 cues. Nat. Rev. Genet. 2012.

667 41. Golicz AA, Batley J, Edwards D. Towards plant pangenomics. Plant Biotechnol J. $668 \quad 2016 ; 14: 1099-105$.

669 42. Montenegro JD, Golicz AA, Bayer PE, Hurgobin B, Lee HT, Chan CKK, et al. The 670 pangenome of hexaploid bread wheat. Plant J. 2017;90:1007-13.

671 43. Li YH, Zhou G, Ma J, Jiang W, Jin LG, Zhang Z, et al. De novo assembly of 672 soybean wild relatives for pan-genome analysis of diversity and agronomic traits. Nat 
673 Biotechnol. 2014;32:1045-52.

674 44. Diamond J. Evolution, consequences and future of plant and animal

675 domestication. Nature. Nature Publishing Group; 2002. p. 700-7.

676 45. Wolko B, Clements JC, Naganowska B, Nelson MN, Yang H. Lupinus. Wild Crop

677 Relat Genomic Breed Resour. Berlin, Heidelberg: Springer Berlin Heidelberg; 2011.

678 p. 153-206.

679 46. Hamblin MT, Jannink J-L. Factors Affecting the Power of Haplotype Markers in 680 Association Studies. Plant Genome J. 2011;4:145.

681 47. Mousavi-Derazmahalleh M, Nevado B, Bayer PE, Filatov DA, Hane JK, Edwards

$682 \mathrm{D}$, et al. The western Mediterranean region provided the founder population of 683 domesticated narrow-leafed lupin. Theor Appl Genet. Springer Berlin Heidelberg; $684 \quad 2018 ; 131: 2543-54$.

685 48. Mousavi-Derazmahalleh M, Bayer PE, Nevado B, Hurgobin B, Filatov D, Kilian A, 686 et al. Exploring the genetic and adaptive diversity of a pan-Mediterranean crop wild 687 relative: narrow-leafed lupin. Theor Appl Genet. Springer Berlin Heidelberg; $688 \quad 2018 ; 131: 887-901$.

689 49. Elias E. Soils of the Ethiopian Highlands: Geomorphology and Properties. 2016.

690 50. Lambers H, Bishop JG, Hopper SD, Laliberté E, Zúñiga-Feest A. Phosphorus691 mobilization ecosystem engineering: the roles of cluster roots and carboxylate 692 exudation in young P-limited ecosystems. Ann Bot. 2012;110:329-48.

693 51. Bolger AM, Lohse M, Usadel B. Trimmomatic: a flexible trimmer for Illumina 694 sequence data. Bioinformatics. 2014/04/01. Oxford University Press; 2014;30:211469520.

696 52. Bankevich A, Nurk S, Antipov D, Gurevich AA, Dvorkin M, Kulikov AS, et al. 697 SPAdes: A New Genome Assembly Algorithm and Its Applications to Single-Cell 
698 Sequencing. J Comput Biol. 2012;19:455-77.

699 53. Hu Z, Sun C, Lu KC, Chu X, Zhao Y, Lu J, et al. EUPAN enables pan-genome 700 studies of a large number of eukaryotic genomes. Bioinformatics. 2017;33:2408-9.

701 54. Camacho C, Coulouris G, Avagyan V, Ma N, Papadopoulos J, Bealer K, et al. 702 BLAST+: architecture and applications. BMC Bioinformatics. BioMed Central; $7032009 ; 10: 421$.

704 55. Yandell M, Holt C. MAKER2: an annotation pipeline and genome-database 705 management tool for second-generation genome projects. BMC Bioinformatics. $706 \quad 2011 ; 12: 491$.

707 56. Stanke M, Morgenstern B. AUGUSTUS: a web server for gene prediction in 708 eukaryotes that allows user-defined constraints. Nucleic Acids Res. 2005;33:W4657097.

710 57. Korf I. Gene finding in novel genomes. BMC Bioinformatics. 2004;5.

711 58. Stanke M, Diekhans M, Baertsch R, Haussler D. Using native and syntenically 712 mapped cDNA alignments to improve de novo gene finding. Bioinformatics. $713 \quad 2008 ; 24: 637-44$.

714 59. Sallet E, Gouzy J, Schiex T. EuGene-PP: A next-generation automated 715 annotation pipeline for prokaryotic genomes. Bioinformatics. 2014;30:2659-61.

716 60. Li H, Durbin R. Fast and accurate long-read alignment with Burrows-Wheeler 717 transform. Bioinformatics. 2010;26:589-95.

718 61. Martin M. Cutadapt removes adapter sequences from high-throughput 719 sequencing reads. EMBnet.journal. 2011;17:10.

720 62. Nguyen L-T, Schmidt HA, von Haeseler A, Minh BQ. IQ-TREE: A Fast and 721 Effective Stochastic Algorithm for Estimating Maximum-Likelihood Phylogenies. Mol 722 Biol Evol. 2015;32:268-74. 
723 63. Kalyaanamoorthy S, Minh BQ, Wong TKF, von Haeseler A, Jermiin LS.

724 ModelFinder: fast model selection for accurate phylogenetic estimates. Nat Methods.

725 Nature Publishing Group, a division of Macmillan Publishers Limited. All Rights

726 Reserved.; 2017;14:587-9.

727 64. Letunic I, Bork P. Interactive tree of life (iTOL) v3: an online tool for the display 728 and annotation of phylogenetic and other trees. Nucleic Acids Res. 2016;44:W242-5.

729 65. Hubisz MJ, Falush D, Stephens M, Pritchard JK. Inferring weak population 730 structure with the assistance of sample group information. Mol Ecol Resour. $731 \quad 2009 ; 9: 1322-32$.

732 66. Zhang C, Dong SS, Xu JY, He WM, Yang TL. PopLDdecay: A fast and effective 733 tool for linkage disequilibrium decay analysis based on variant call format files.

734 Bioinformatics. 2019;35:1786-8.

735 67. Bradbury PJ, Zhang Z, Kroon DE, Casstevens TM, Ramdoss Y, Buckler ES. 736 TASSEL: software for association mapping of complex traits in diverse samples. 737 Bioinformatics. 2007;23:2633-5.

738 68. Pfeifer B, Wittelsbürger U, Ramos-Onsins SE, Lercher MJ. PopGenome: An 739 Efficient Swiss Army Knife for Population Genomic Analyses in R. Mol Biol Evol. $740 \quad 2014 ; 31: 1929-36$.

\section{ACKNOWLEDGEMENTS}

742

743 DECLARATIONS

\section{Ethics approval and consent to participate}

745 Not applicable

746

747 Consent for publication 
748 Not applicable

749

\section{Competing interests}

751 The authors declare that they have no competing interests.

752

\section{Funding}

754 This project has received funding from the European Research Council (ERC) under 755 the European Union's Horizon 2020 research and innovation program (Starting Grant 756 LUPINROOTS - grant agreement No 637420 to B.P.) and from the Innovate UK 757 project 133048 (Ethiopian Lupins for Food and Feed) to H.S.

758

\section{Availability of data and materials}

760 The detailed methods and datasets supporting the conclusions of this report are

761 included within the article and its additional files. All deep sequencing data reported 762 in this paper have been submitted to the NCBI. The datasets generated and 763 analyzed during the current study are available from the corresponding author upon

764 request. Full genomic and raw sequence data are publicly available for download on

765 the White Lupin genome portal [www.whitelupin.fr/pangenome] that contains a

766 Genome Browser, Expression tools and a Sequence retriever dedicated to the 767 pangenome. The pangenome project and raw data has been deposited at 768 DDBJ/ENA/GenBank under the accession PRJNA608889.

769

770 Authors' contributions

771 A.S. developed bioinformatic resources and performed pangenome assembly. J.T.

772 and F.D. performed DNA extraction and experiments. M.N., H.S., L.Y. and M.K. 
bioRxiv preprint doi: https://doi.org/10.1101/2020.06.21.163378; this version posted June 22, 2020. The copyright holder for this preprint (which was not certified by peer review) is the author/funder, who has granted bioRxiv a license to display the preprint in perpetuity. It is made available under aCC-BY-ND 4.0 International license.

773 provided genetic material. B.H. performed data analysis. B.H., M.K., M.N. and B.P.

774 designed experiments and wrote the article.

775

776 Corresponding authors

777 Correspondence to Benjamin Péret (benjamin.peret@supagro.fr) and Bárbara

778 Hufnagel (barbara.hufnagel@supagro.fr). 
a

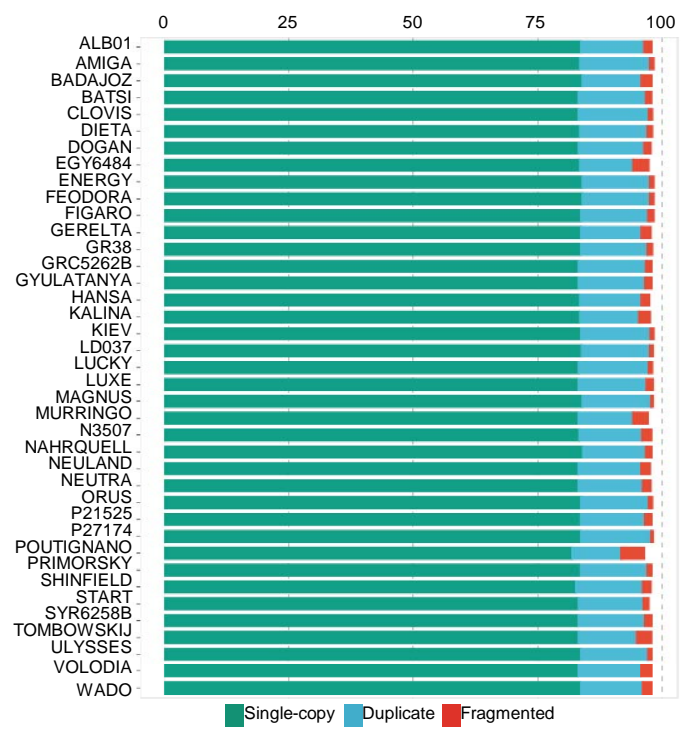

b

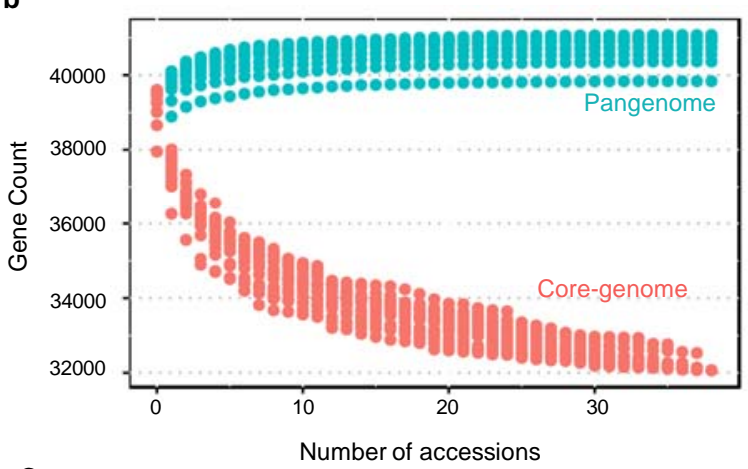

C

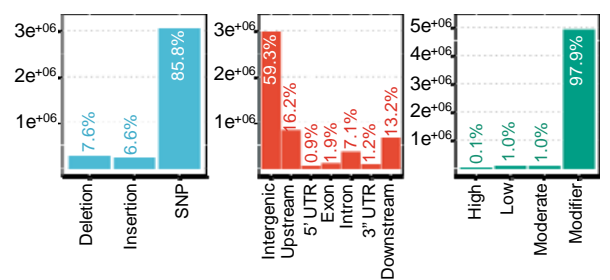

Figure 1. Pangenome of L. albus. (a) BUSCO percent completeness of all assemblies. All of the assemblies of this study have BUSCO completeness higher than $91.7 \%$. (b) Pangenome modeling (c) Distribution of variants along white lupin pangenome. Types of variations identified (blue); positioning of the variants in the genome in relation to the gene structures (red); impact of the variants (green). 
a

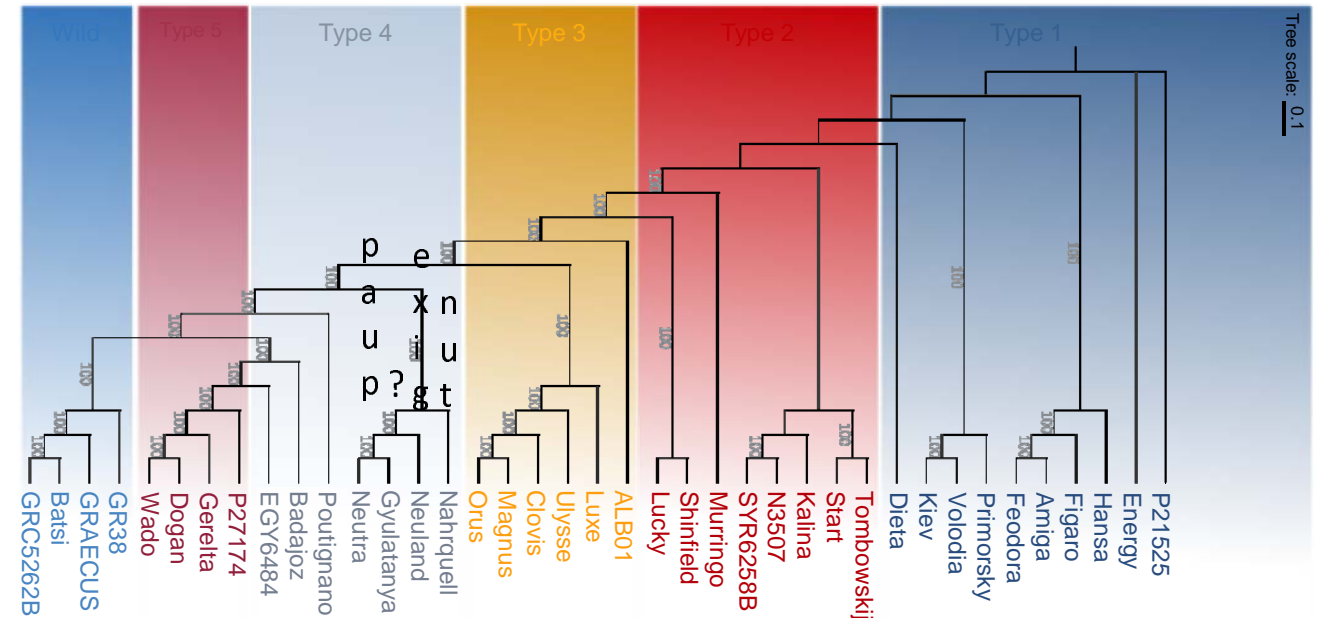

b

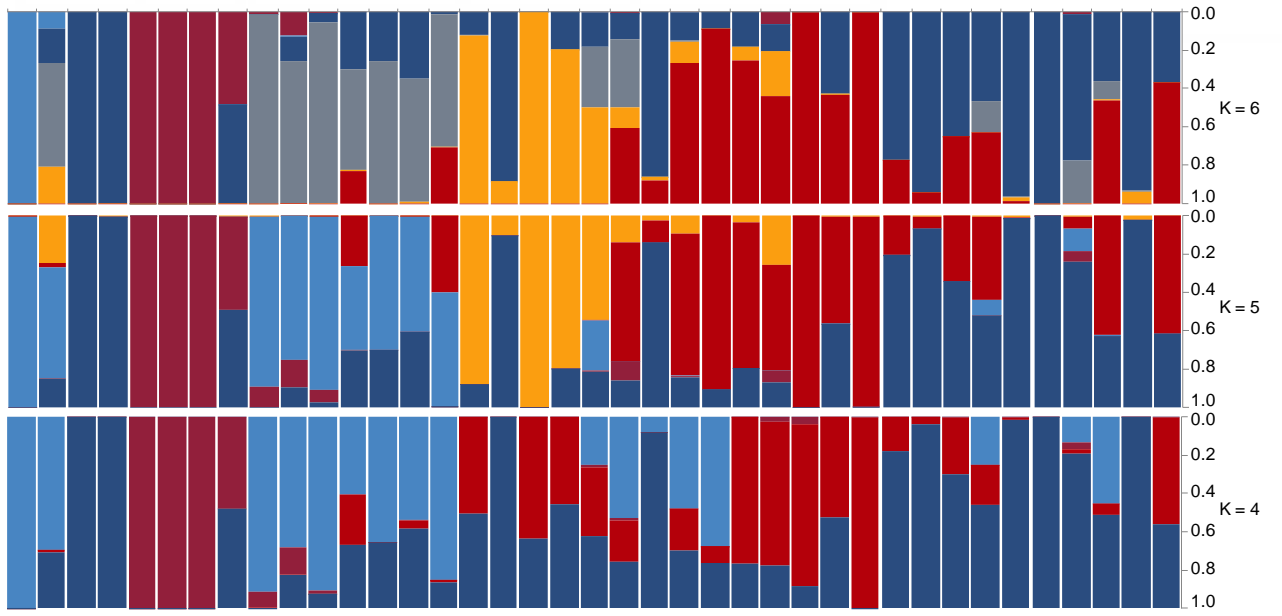

c
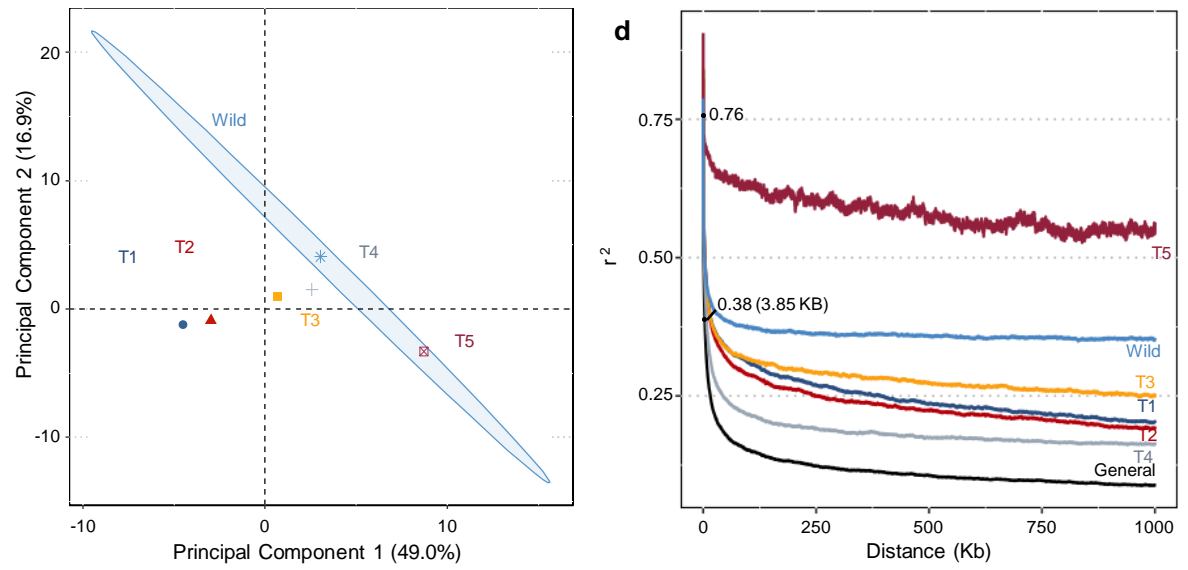

Figure 2. Phylogeny and population structure of 39 accessions of $L$. albus. (a) Maximum likelihood phylogenetic tree of white lupin constructed based on 3.5 M SNPs. The accessions are divided in 6 idiotypes. (b) Model-based clustering analysis with different numbers of ancestral kinships ( $k=4,5$ and 6$)$. The y axis quantifies cluster membership and the $x$ axis list the different accessions. The positions of these accessions on the $x$ axis are consistent with those in the phylogenetic tree. (c) Principal component analysis based on 3.5 M SNPs. The ellipses are discriminating the accessions of each idiotype groups. (d) Genome-wide average LD decay estimated from different white lupin group. The decay of LD with physical distance between SNPs to half of the maximum values occurred at $3.85 \mathrm{~kb}\left(\mathrm{r}^{2}=0.38\right)$ considering all accessions. 
a

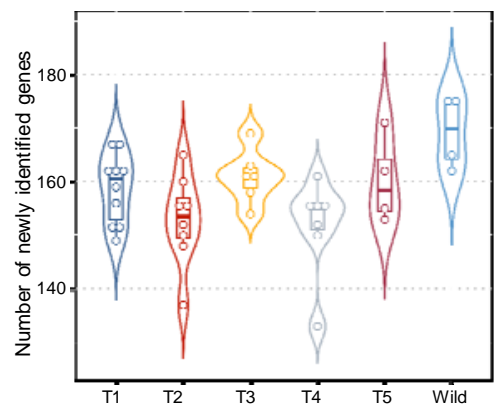

b

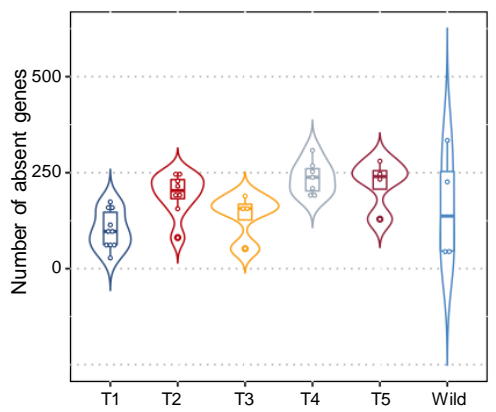

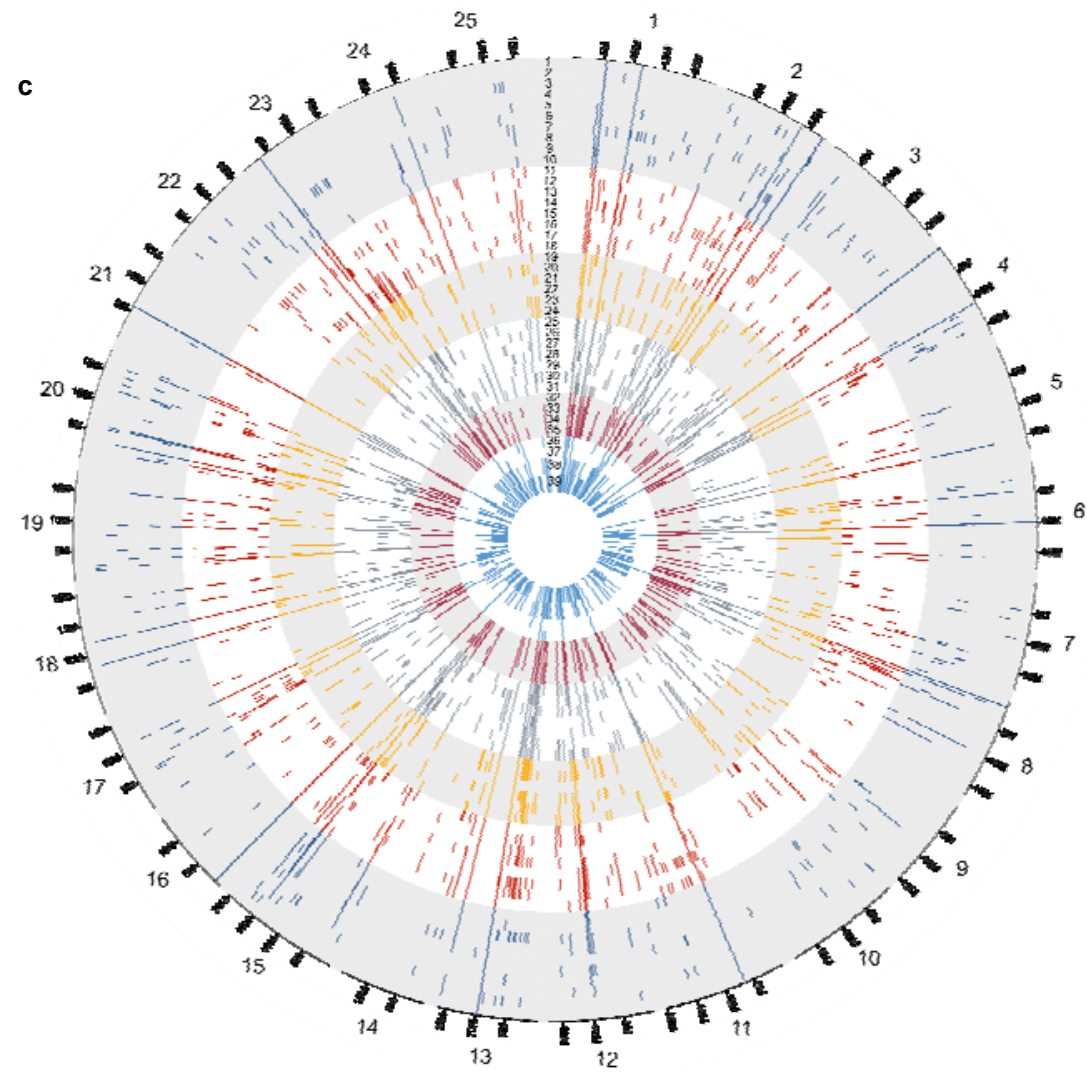

d

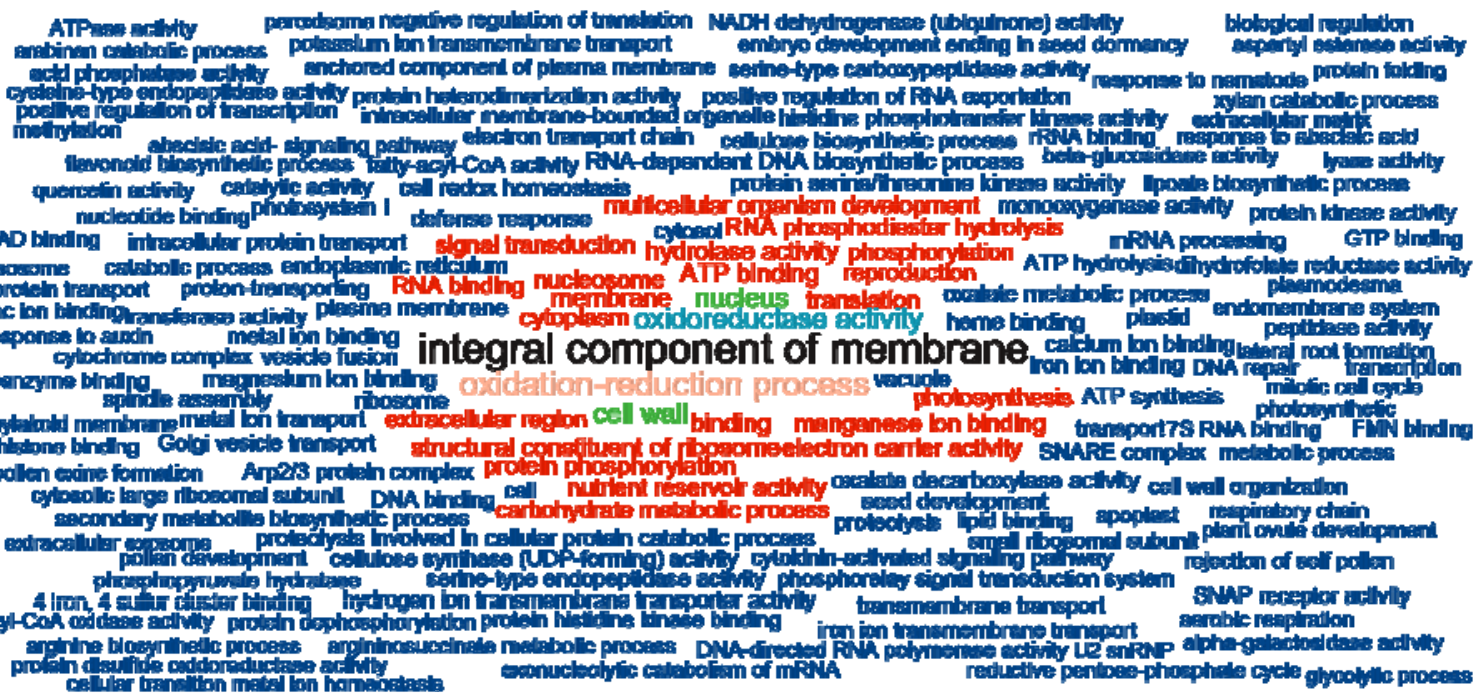

Figure 3. PAV of coding gene in L. albus. (a) Number of newly identified genes by phylogenetic group. (b) Number of absent genes by phylogenetic groups. (c) Positioning of absent genes in the 25 white lupin chromosomes in each one of the 39 accessions. Order of accessions from outer to inner track: 1-AMIGA, 2-FEODORA, 3-FIGARO, 4ENERGY, 5-KIEV MUTANT, 6-HANSA, 7-P21525, 8-PRIMORSKY, 9-DIETA, 10VOLODIA, 11-START, 12-N3507, 13-TOMBOWSKIJ, 14-KALINA, 15-SYR6258B, 16LUCKY, 17-MURRINGO, 18-SHINFIELD, 19-ALB01, 20-LUXE, 21-ULYSSE, $22-$ MAGNUS, 23-CLOVIS, 24-ORUS, 25-NAHRQUELL, 26-GYUNLATANYA, 27NEULAND, 28-NEUTRA, 29-BADAJOZ, 30-EGY6484B, 31-POUTIGANO, 32-P27174, 33-GERELTA, 34-DOGAN, 35-WADO, 36-GR38, 37-GRAECUS, 38-BATSI, 39GRC5262B. The accessions' colors reflect the 6 idiotypes. (d) Functional enrichment analysis of the variable genome. Graphical representation of enriched biological process (GOs). Size of the words and colors are proportional to their representativeness in the aene pool. 

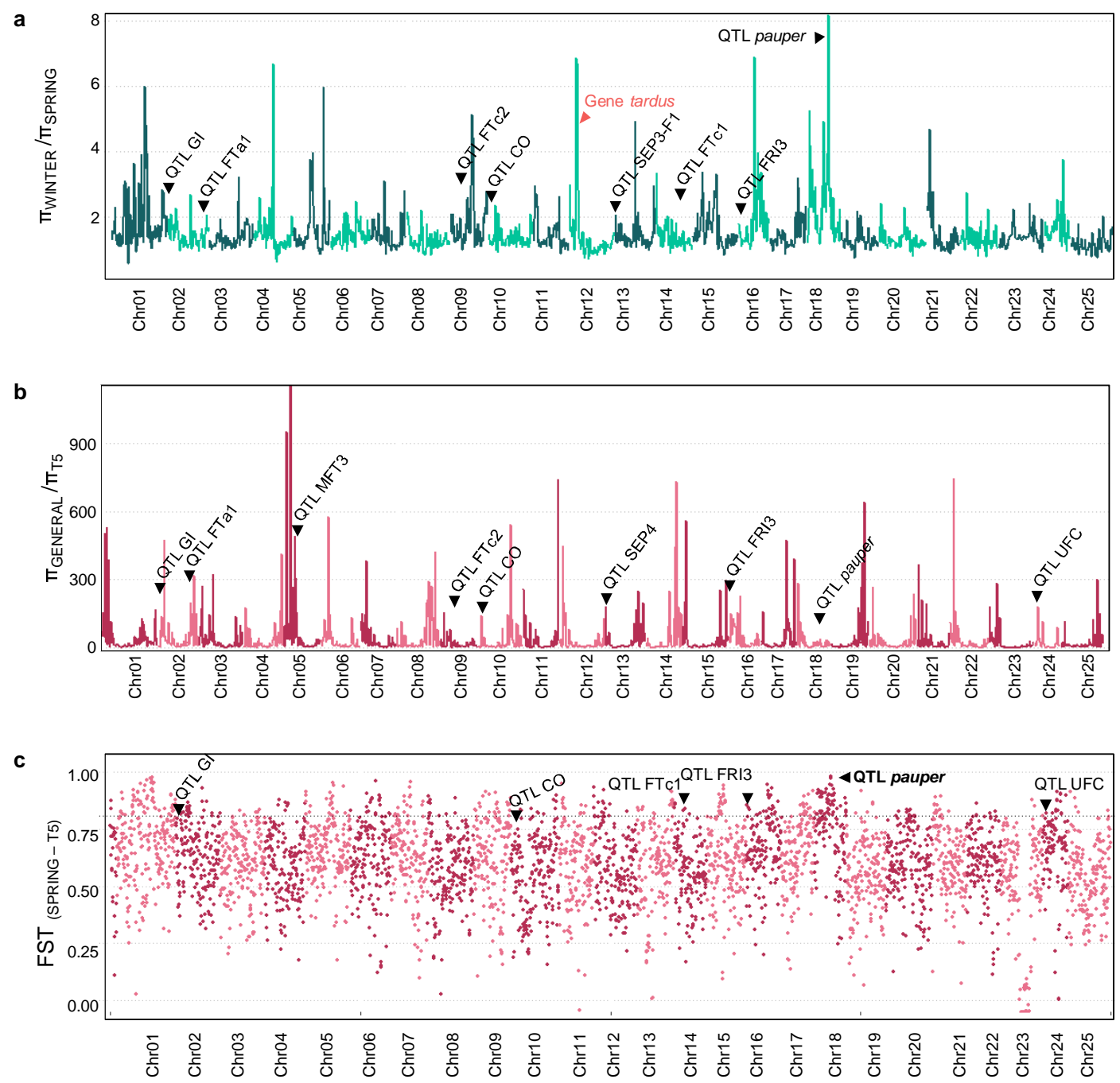

Figure 4. Footprints of selection in the white lupin genome. Nucleotide diversity ( $\pi$ ) comparison between (a) Winter (T3 and T4) and Spring accessions (T1 and T2) and (b) between all accessions (General) and Ethiopian accessions (T5). QTLs previously reported and a $L$. angustifolius domestication gene (red) that overlapped with selective sweeps are marked. (c) Fst-based genome-wide analysis of population differentiation estimated between Spring (T1 and T2) and Ethiopian (T5) accessions. Black horizontal dashed line marks the .90 percentile of distribution of Fst estimated ( $F s t=0.81)$. 
a

FLOWERING LOCUS T-LaFTa1

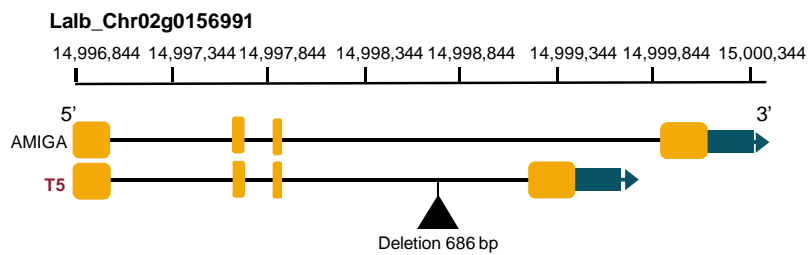

b

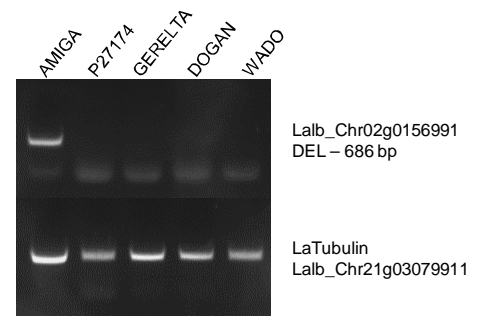

Figure 5. Identification and allele variation of candidate gene FLOWERING LOCUS T. (a) Candidate gene located on chromosome 2. Type 5 accessions, originated from Ethiopia, have a deletion of $686 \mathrm{bp}$ in the third intron. (b) Confirmation of the deletion in the third intron of Type 5 accession by PCR. Gene LaTubulin was used as positive control. 